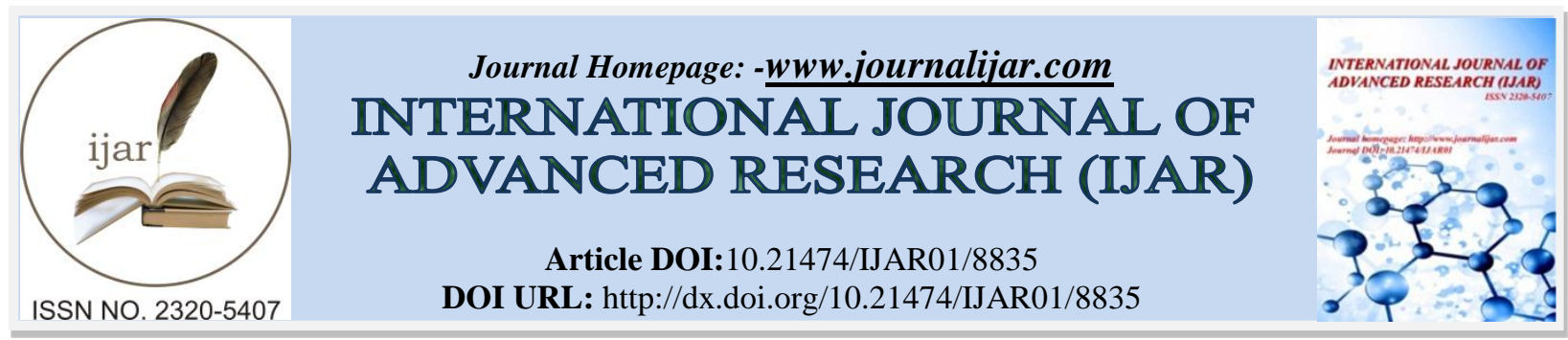

RESEARCH ARTICLE

\title{
A NOVEL APPROACH IN PATIENT CARE AND NON-PHARMACOLOGICAL TREATMENT FOR RHEUMATOID ARTHRITIS.
}

Athira. PV and Shabaraya AR.

Department of Pharmacy Practice, Srinivas College Of Pharmacy, Valachil, Manglore.

\section{Manuscript Info}

\section{Manuscript History}

Received: 07 February 2019

Final Accepted: 09 March 2019

Published: April 2019

\section{Key words:-}

Rheumatoid arthritis, aggressive therapy, non-pharmacological treatment, patient care.

\begin{abstract}
Rheumatoid Arthritis (RA) is a chronic, systemic autoimmune disease characterized by persistent inflammation of synovial joints, often leading to joint destruction and disability. The major goals of treatment are to relieve pain, reduce inflammation, slow down or stop joint damage, prevent disability and preserve or improve the patients sense of wellbeing and ability to work. ${ }^{[1]}$

RA, a chronic disease affecting $0.5 \%-1 \%$ of adults, is characterized by persistent synovitis, systemic inflammation, and immunological abnormalities.
\end{abstract}

Copy Right, IJAR, 2019,. All rights reserved.

\section{Introduction:-}

Uncontrolled RA causes damage to the joints, disability, and decreased quality of life along with other comorbidities. The environment and gene interactions leads to loss of tolerance to self-antigens that contain a citrulline residue generated by post-translational modification, leading to an anticitrulline response by both T-cells and B-cells. Thereafter, the inflammatory response becomes localized in the joints and synovitis and further leads to systemic disorders. The lymphocytes, other inflammatory cells and their products contribute to the development of RA. For example, many cytokines have been implicated in the pathogenesis of RA, including tumor necrosis factor (TNF)- $\alpha$, interleukin (IL)-1, IL-6, IL-7, IL-15, IL-17A, IL-17F, IL-18, IL-21, IL-23, IL-32, IL-33, and granulocytemacrophage colony stimulating factor. ${ }^{[2]}$

\section{Current Framework Of Rheumatoid Arthritis Therapy}

The two concepts that guide early RA treatment are early aggressive therapy and treat to target ( $\mathrm{T}$ to $\mathrm{T}$ ) . Early aggressive therapy is the prompt institution of agents to decrease inflammation and thereby prevent the joint destruction that leads to pain and disability. They act by reducing inflammation by blocking immune mediators or by modulating the number or functional properties of immune cells. These agents are known as disease modifying antirheumatic drugs (DMARDs). Methotrexate and TNF blockers are 2 of the most commonly used agents. Many others molecules which target new mediators and pathways are in developing process. ${ }^{[3]}$

For any disease, the administration of optimal therapy depends upon achieving a target. Frequently, the target is a laboratory value such as glucose or hemoglobin A1C, although a physiological measure can also be a target (eg, blood pressure). For RA, the target is disease activity. ${ }^{[4]}$

Corresponding Author:-Athira. PV.

Address:-Department of Pharmacy Practice, Srinivas College Of Pharmacy,Valachil, Manglore. 


\section{Early Diagnosis And Treatment}

Early diagnosis and treatment are the key to improve RA outcomes which is not usually achieved. Patients may selfmedicate and the time to seek medical advice may be crossed. At this point the disease should have progressed to the stage of damage. Education to the public regarding the disease should be given by conducting camps and awareness programs. Similarly professional education and proper knowledge regarding the disease is essential for a better outcome in the treatment.

At present there is a severe shortage of rheumatologists and other health professionals with specialized training in RA. This shortage is worldwide and the access may be limited due to a many factors like significant delays in obtaining a referral. The solution to this problem is to development of better health care systems and to put up early arthritis clinics. In Europe, early arthritis clinics have been in operation from many years .Not surprisingly, European investigators have been in the forefront of research on the diagnosis and treatment of early RA and the development of early aggressive therapy and $\mathrm{T}$ to $\mathrm{T} .{ }^{[5]}$

\section{Treat To Target}

Early aggressive therapy with $\mathrm{T}$ to $\mathrm{T}$ approach is designed to reduce inflammation, prevent damage, and to limit late complications. Many clinical studies show that numerous agents alone or in combination are used currently to achieve this goal. An agent to rapidly reduce inflammation is an important element in early aggressive therapy and provides a beginning for other DMARDs to act ${ }^{[6]}$. Methotrexate is used as the anchor drug for most patients for this purpose. Glucocorticoids at high doses are also frequently used to achieve this though these agents show significant toxicity and are not well tolerated by patients, especially those with comorbidities such as diabetes or hypertension. Another approach to early aggressive therapy involves the use of biologic therapy, specifically tumor necrosis factor (TNF) blockers, in combination with methotrexate. TNF blocking agents blocks the cytokine and it has many immunological effects, including a prompt reduction in inflammation, instantaneous pain relief, increased patient energy and well-being. TNF blockers are considered for monotherapy or in association with methotrexate. The major difference between glucocorticoids in combination with methotrexate or combination of TNF blocker and methotrexate is costs. ${ }^{[7]}$.

Many rheumatologists begin with an initial therapy with a TNF blocker along with methotrexate represents one of the best current approaches to RA therapy. The side effect profile is also tolerable. Here cost considerations does not limit the use of this combination. ${ }^{[3]}$

American College of Rheumatology (ACR) and European League Against Rheumatism (EULAR) developed new classification criteria for RA in 2010 aimed at early diagnosis . These criteria are intended for patients with at least 1 joint with definite clinical synovitis symptoms like swelling, not just tenderness and in whom the synovitis is not explained by another disease such as psoriasis, systemic lupus erythematosus, or gout. Patients are considered to have RA only if they have a score of at least $6 .{ }^{[8]}$

\section{Recent Advancement In The Treatment Of Ra}

The recent advancement in pharmacologic therapy has led to improved outcomes and remission is now possible for many patients. These advances are obtained from increased scientific understanding of the disease as well as the development of new therapeutic agents that more specifically target important disease mediators. Among these agents, the biologics have produced dramatic results in well-designed clinical trials. Biologics are protein molecules and include monoclonal antibodies like infliximab and soluble receptors like etanercept ${ }^{[3]}$.

While clinical outcomes have improved, the costs of care for RA and other forms of inflammatory disease demand consideration of strategies to allocate resources more efficiently and effectively. In some instances, these approaches focus on allocation of medical personnel and in other instances on refinement in medication use. ${ }^{[3]}$

The biologic therapies have changed the way RA is managed and it is well validated. These targeted specific therapies acts on the cytokines and its receptors and modifies immune mediated damage. Potential targets in RA are cytokines for example TNFa, IL1 and IL6, B cells, molecules that leads to interaction between antigen presenting cells and T cells, receptor activator of nuclear factor kB ligand (RANK ligand) and intracellular signal mediating small molecules. At present FDA have approved nine biologics for the treatment of RA and they are TNF inhibitors for example infliximab, etanercept, adalimumab, certolizumab pegol, and golimumab, anakinra which is an IL-1 
receptor antagonist, abatacept which is a CTLA4-Ig fusion protein, rituximab which is an antiCD20 antibody, and tocilizumab which is an anti-IL-6 receptor antibody. For those patients who have at least one failed treatment with TNF inhibitor ,Rituximab and tocilizumab are currently used which is approved for RA patients. ${ }^{[8]}$

A large number of monoclonal antibodies along with its bio similar molecules are available worldwide including India. Though the safety and efficacy of each molecule have been evaluated before study, pharmacovigilance is required to identify any ADRs. On evaluation of the ADRs reported in the NCC_PvPI from April 2011 to February 2014 the monoclonal antibodies showed the highest number of ADR which were fatal and serious . ${ }^{[9]}$

Although biologic tapering trials are performed, adequate number of studies must be done to obtain a guide to the clinicians with regard to RA treatment.$^{[10]}$

\section{Non-pharmcological treatment for ra:}

The management of RA is incomplete without the non-pharmacological management. A wide range of nonpharmacological treatments along with its guidance are available at various RA clinics or health care centers. They are occupational therapy (OT), balneotherapy which is bathing in hot mineralised water, either with minerals added or in naturally occurring hot springs, tai chi, acupuncture, and low level laser therapy. Most of the patients may require a long term patient care along with patient education guidance and support to cope up with the disease and daily activities.

Each patient will be guided for each non-pharmacological therapy based on the patient condition, financial support along with the patient preferences. The team members that are involved in providing health care to RA patients are the rheumatologist, nurse specialists, physical therapists, occupational therapists, social workers, dieticians, podiatrists, psychologists, general practitioners, orthopedic surgeons and rehabilitation specialists and they are known as the multidisciplinary rheumatology team ${ }^{[11]}$. The exercises will be planned and designed according to the factors that affect the daily activities and these will be performed in a repetitive manner so as to improve the physical fitness ${ }^{[12]}$. The aims of these exercises are to improve the joint movements, muscle strength and performance of daily activities ${ }^{[13] .}$

1. Thermotherapy is the one in which either ice or heat is used as a therapeutic intervention. Local cold compression with ice packs, ice chips or ice massage and heat compression with hot packs, paraffin or wax baths, thermal baths and infrared deep heat methods like electromagnetic wave forms and ultrasound are used. These are used to overcome stiffness and relieve pain ${ }^{[14]}$.

2. Electrotherapy is mainly done to control pain and for muscle stimulation and it is applied with the help of surface electrodes are used. It uses different forms of electric currents.

3. Low level laser therapy is a method by which pure light is generated with a single wavelength ${ }^{[11]}$.

4. Balneotherapy is the one in which water from mineral and thermal springs has been particularly used and a spa therapy with temperatures of $34-38 \mathrm{C}$ for a duration of about 20 minute is done. ${ }^{[15]}$

5. Joint protection and energy conservation techniques like Aids, devices and adaptations to physical environment for example jar openers, raised toilets seats, bathroom appliances and special beds ${ }^{[16]}$.

6. Orthoses are those medical devices that are underpinned to a person's body to align, support, position, immobilize, prevent or correct deformity, assist weak muscles or improve function

7. Special shoes and inserts are available which helps in reducing shock and shear and provide further support ${ }^{[17]}$.

8. Cognitive Behavioral Therapy (CBT) and other psychological therapies were developed to target mood issues and chronic pain in adults. CBT is a structured self-management intervention that teaches a blend of cognitive and behavioral management.

9. Physical Activity and Exercise are recommended on a daily basis. The American College of Rheumatology and the American Pain Society recommend aerobic exercise and physical therapy .The three main types of exercises: (a) flexibility and range-of-motion (b) muscle conditioning and resistance training and (c) aerobic exercise $^{[18]}$

\section{Patient education and counseling :}

Education and counseling are important in the management of rheumatoid arthritis as many patients have misconceptions about the nature of arthritis and its cause. Providing the right information may help establish a good long-term relationship between the clinician and patient ${ }^{[19]}$. 
European Rheumatologic Guidelines underline the correct principle to share decisions between patient and rheumatologist ${ }^{[20]}$.

\section{Patient care in rheumatoid arthritis:}

The impact of the disease is distinguished and defined by the World Health Organization into the following levels impairment, disability and handicap.

Impairment refers to loss or abnormality of psychological or anatomical structure or function. Disability is any restriction or an activity limitation or difficulty encountered by an individual in executing a task or action. Handicap is a condition that markedly restricts their ability to function physically, mentally and socially ${ }^{[21]}$.

The European Union commission [EU] and European League against Rheumatism [EULAR] has done a study for the development of patient centered standards of care for rheumatoid arthritis in Europe. This team has focused in the development of set of recommendations for European Standards of Care and has introduced a checklist for the patients for the patients. The Standards of care are developed to apply for all European countries and should be implemented by 2020 .

This ensures that a large rheumatoid arthritis patient population is obtaining standard patient care. This is the first study done for developing the standards of care and it also helps in reducing any inequalities in treatment across Europe by providing evidence based information ${ }^{[22]}$.

Those patients who have consulted the rheumatoid specialist during their treatment period have shown a better outcome and have also received higher quality of care compared to others who have consulted a primary physician or primary health care provider.

Though all dimensions of quality cannot be covered, the study has covered a range of dimensions of quality and was marked by certain codes. Efforts should be taken to improve the physician knowledge and patient awareness with regard to this chronic disease ${ }^{[23]}$.

The recent studies show that the quality of care should be considered from the perspective of patients. The indicator to quality of care is patient satisfaction.

Netherlands have developed an instrument, the QUOTE- questionnaire which incorporates the dimensions of international literature and this helps in proper rating of quality. However the quality of care should be rated only with a discussion with patients as there are chances of bias in terms of age, education, marital status, gender, and other health characteristics.

The study showed that expect the rheumatologist, other health care providers had inadequate information of rheumatology and the patients had noted this condition. It is high time to conduct educational programs and awareness programs to improve the knowledge and skills of the health care professionals regarding rheumatoid arthritis. The high expectations from the patient side from the health care may be another aspect that led to the conclusion regarding inadequate information with the physician. The educational programs have helped to overcome such thoughts in the patients ${ }^{[24]}$.

Since the clinicians can afford only few hours with the patients, it is necessary for the patients to be actively involved in self-care and management. To promote the above, following are important:

1. Patient education,

2. patient involvement or shared decision-making,

3. patient empowerment or self-management,

4. involvement of family and friends and

5. physical and emotional support ${ }^{[25]}$.

The active patient involvement in healthcare is essential crucial to achieve common standards of care in various countries. Certain factors support this with the existence of active patient organizations, the persistence of traditional models of healthcare, the existence of patient experts in advisory boards of research projects and inhibit it by nonphysician health professionals taking on extended roles ${ }^{[26]}$. 
Rheumatoid arthritis which is a chronic inflammatory disease, care, management and cost varies co among different rheumatologists. The reason for this variability in term of management and both cost have not been understood clearly yet. This particular study has explained the reason for variability in care and cost. The main and only reason for increase in cost was due to the unnecessary tests and use of biologic agents. There still lies an opportunity to overcome these additional costs and improve quality of treatment and care ${ }^{[27]}$.

\section{References:-}

1. Shaw K, Zochling J, Winzenberg T.Nonpharmacological interventions for rheumatoid arthritis.Australian Family Physician. 2007 oct;36(10):840-1.

2. Tanaka T, Hishitani Y, Ogata A.Monoclonal antibodies in rheumatoid arthritis: Comparative effectiveness of tocilizumab with tumor necrosis factor inhibitors. Dove Press.2014 April;8(7);141-2.

3. Smolen JS, Aletaha D, McInnes IB. Rheumatoid arthritis. Lancet. 2016;388(10055):2023-2038.

4. Felson DT, Smolen JS, Wells G, Zhang B, van Tuyl LH, Funovits J, et al. American College of Rheumatology/European League Against Rheumatism provisional definition of remission in rheumatoid arthritis for clinical trials. Arthritis Rheum. 2011;63(3):573-586.

5. David S. Pisetsky.Advances in the treatment of Rheumatoid Arthritis.NCMJ.2017;78(5):337-9.

6. Nam JL, Villeneuve E, Hensor EM, Conaghan PG, Keen HI, Buch MH, et al. Remission induction comparing infliximab and high-dose intravenous steroid, followed by treat-to-target: a double-blind, randomised, controlled trial in new-onset, treatment-naive, rheumatoid arthritis (the IDEA study). Ann Rheum Dis. 2014;73(1):75-85.

7. Jalal H, O’Dell JR, Bridges SL, Jr., Cofield S, Curtis JR, Mikuls TR, et al. Cost-effectiveness of triple therapy versus etanercept plus methotrexate in early aggressive rheumatoid arthritis. Arthritis Care Res (Hoboken). 2016;68(12):1751-1757.

8. Joshi M.Advances in the management of Rheumatoid Arthritis.J MGIMS.2012;17(2):6-12.

9. Kalaivani M, Abhishank, V Kalaiselvan.Therapeutic monoclonal antibodies and need for targeted Phamacovigilance in India.mAbs.2015;7(1):276-80.

10. Mahajan TD, Mikuls TR.Recent advances in the treatment of Rheumatoid Arthritis.curr opin Rheumatol.2018 May;30(3):231-37.

11. Vlieland VTPM.Non-drug care for RA-is the era of evidence-based practice approaching?Rheumatology.2007 June;149(46):1397-1404.

12. Caspersen CJ, Powell KE, Christenson GM. Physical activity, exercise, and physical fitness: definitions and distinctions for health-related research. Public Health Rep 1985;100:126-31.

13. Vlieland VTPM. Rehabilitation of people with rheumatoid arthritis. Clin Rheumatol (Best Pract Res $\mathrm{Cl} \mathrm{Rh}$ ) 2003; 17:847-61.

14. Oosterveld FGJ, Rasker JJ. Treating arthritis with locally applied heat or cold. Semin Arthritis Rheum 1994;24:1-10.

15. Van Tubergen A, van der Linden S. A brief history of spa therapy. Ann Rheum Dis 2002;61:273-5.

16. Heide A, Jacobs JWG, van Albada-Kuipers GA, Kraaimaat FW, Geenen R, Bijlsma JWJ. Self report functional disability scores and the use of devices: two distinct aspects of physical function in rheumatoid arthritis. Ann Rheum Dis 1993;52:497-502.

17. Janisse DJ. Prescription footwear for arthritis of the foot and ankle. Clin Orthop Relat Res 1998;349:100-7.

18. Cunningham RN, Zuck SK.Nonpharmacological treatment of pain in Rheumatic diseases and other Musculoskeletal pain conditions.curr opin Rheumatol.2013 February;15(2):306.

19. Schur PH, Gibofsky A,James R O'Dell,Romain Pl.nonpharmacologic therapies and preventive measures for patients with rheumatoid arthritis.uptodate;2018 July.

20. Donzelli A, schivalocchi A. lifestyle and rheumatoid arthritis:prevention and non-pharmacological treatment.JVASC.2016;2(4):9544.100117.

21. Carr AJ.A patient Centered Approach To Evaluation and Treatment in Rheumatoid Arthritis: The Development of a clinical tool to measure patient perceived handicap.British Journal of Rheumatology.1996;35:921-932.

22. Stoffer MA, Smolen JS, Woolf A, Ambrzic A, Baswort A, Carmone L et al.Development of patient centred standards of patient centred standards of care for Rheumatoid Arthritis in Europe: the eumusc.netproject.Ann Rheum Dis.2013;20141(73):902-905.

23. Catherine H, Maclean, Louie R, Leake B.Quality of care for Patients with Rheumatoid Arthritis.American Medical Association.2018 dec;2000(284):984-921.

24. Jacobi CE, Boshuizen HC, Rupp I, Huibert J Dinant Heertruidis A M Van Den Bos.Quality of rheumatoid arthritis Care:The patient's perspective .International Journal for Quality in Healthcare.2014 Nov;16(1):73-81. 
25. Voshaar MJH, Nota I, Van De MAFJ.Patient centered care in established rheumatoid arthritis.doi.2015 sept:007.

26. Stamm T, Stoffer M, wit M, Redlich K, Woolf A, Smolen J.Putting patients first:the need for evidence based and patient centered standards of care in rheumatoid arthritis.Future Medicine.2014;9(1);1-2.

27. Maria D, Acelajado MC, Luck J, Ta H,Chernoff D, Florentino J, Peabody JW.Variations and practice in the care of patients with rheumatoid arthritis:Quality and cost of care.J Clin Rheumatol.2014 march;20(2);79-86. 